\title{
Inertial acceleration as a measure of linear vection: An alternative to magnitude estimation
}

\author{
THEODORE R. CARPENTER-SMITH, ROBERT G. FUTAMURA, and DONALD E. PARKER \\ Miami University, Oxford, Ohio
}

\begin{abstract}
The present study focused on the development of a procedure to assess perceived self-motion induced by visual surround motion-vection. Using an apparatus that permitted independent control of visual and inertial stimuli, prone observers were translated along their head $x$-axis (fore/aft). The observers' task was to report the direction of self-motion during passive forward and backward translations of their bodies coupled with exposure to various visual surround conditions. The proportion of "forward" responses was used to calculate each observer's point of subjective equality (PSE) for each surround condition. The results showed that the moving visual stimulus produced a significant shift in the PSE when data from the moving surround condition were compared with the stationary surround and no-vision condition. Further, the results indicated that vection increased monotonically with surround velocities between 4 and $40 \%$ sec. It was concluded that linear vection can be measured in terms of changes in the amplitude of whole-body inertial acceleration required to elicit equivalent numbers of "forward" and "backward" self-motion reports.
\end{abstract}

The movement of an observer through the environment is typically a multisensory process involving visual, vestibular, and somatosensory integration. However, movement of the observer's visual surround is a sufficient stimulus for self-motion perception. The interpretation of visual surround motion as self-motion is supported by the fact that motion of the entire visual surround typically results from motion of the head (body) in space. Further, vestibular receptors are designed to transduce only accelerations of the head (body) and therefore yield the same response during body rest and constantvelocity self-motion.

The earliest investigations of self-motion induced by moving visual stimuli involved stationary observers seated in large rotating or translating visual surrounds (Mach, 1875). Observers in these situations reported self-motion with respect to a stationary environment. This visually induced self-motion perception was subsequently labeled vection (Fischer \& Kornmuller, 1930). Most vection research has involved stationary observers seated in a rotating black and white striped cylinder, and

This research was supported by Grants NGT-50427 and NAG 9446/Basic from the National Aeronautics and Space Administration to Miami University. This study was the Miami University doctoral thesis of T. Carpenter-Smith. We would like to thank Allan Benson, Allan Pantle, and Walter Bisch of for their contributions to the psychophysical methods and analysis. The comments of Richard Held and the anonymous reviewers on an earlier draft of this paper are gratefully acknowledged. Correspondence should be addressed to T. CarpenterSmith, Man-Vehicle Laboratory, Department of Aeronautics and Astronautics, Massachusetts Institute of Technology, Cambridge, MA 02139 (e-mail: tcs@space.mit.edu). the self-motion perception that arises is referred to as circular vection. The circular vection literature has been reviewed by Dichgans and Brandt (1978) and more recently by Howard $(1986,1989)$. Linear vection is elicited by exposing stationary observers to a visual display that expands, contracts, or moves unidirectionally in the frontal or lateral field of view. Linear vection has been reviewed by Dichgans and Brandt (1978) and Berthoz and Droulez (1982).

In order to investigate the role of vision in self-motion perception, several dependent measures have been employed. These measures include subjective estimates, postural adjustments, and judgments of heading or direction (Warren \& Kurtz, 1992). Another measure, not often cited, is the extent to which a moving visual surround interferes with an observer's ability to "null" perturbations in his or her inertial self-motion (Huang \& Young, 1981, 1987; Zacharias \& Young. 1981). The nulling paradigm is described below. All of these measures are designed to reveal the presence or absence of vection, but only postural sway and subjective estimation have been used to investigate the quantitative relationship between surround motion magnitude and vection.

\section{Postural Sway}

An observer standing on a stationary platform will sway in response to motion of the visual surround. It is not clear whether postural sway induced by a moving visual surround is a measure of the perceived self-motion or change of self-orientation with respect to the visual vertical (see Dichgans \& Brandt, 1978). For example. during exposure to constant-velocity visual motion, dis- 
turbances in posture are greatest at visual surround motion onset and attenuate while reports of self-motion perception persist (Lestienne, Soechting, \& Berthoz, 1977). However, sinusoidal variations in visual surround motion elicit continuous postural sway of approximately equal magnitude across the entire exposure period (Andersen \& Dyre, 1989; Lee \& Aronson, 1974; Stoffregen, 1985). Taken together, these findings suggest that accelerated rather than constant-velocity visual motion is the stimulus to the posture control system. Therefore, the usefulness of postural sway may be in understanding the perception of visual acceleration.

\section{Subjective Estimation}

Most vection researchers have used the method of magnitude estimation. This method assumes that an observer is able to indicate the magnitude of his/her selfmotion perception by displacing a joystick or by generating a numerical estimate to indicate perceptual intensity. A more detailed discussion of magnitude estimation can be found elsewhere (Engen, 1972; Poulton, 1968; Stevens, 1961). The application of magnitude estimation to vection research offers several disadvantages, some of which relate to general criticisms of the method (Brindley, 1970; Poulton, 1968; Teghtsoonian, 1971).

Magnitude estimation, as employed in vection research, requires that an observer be able to scale his/her perceptions with respect to a stable modulus or reference stimulus level. However, it is known that vection is characterized by short-term and possibly long-term adaptation (Berthoz \& Droulez, 1982; Schmidt \& Tiffin, 1969). Therefore, the experimenter is left unable to discriminate between an adapted response and the possibility that the observer has difficulty recalling the perception of the modulus when it is presented during subsequent exposures.

A second limitation of self-motion magnitude estimates is related to the format of the data. One significant advantage of other reporting methods (e.g., threshold determination, matching tasks, and signal detection paradigms) is that the data obtained are directly comparable between observers. Standard convention in magnitude estimation is to normalize the estimates to eliminate the influence of differences in estimate range adopted by the observers (Engen, 1972). The resulting comparison between observers is, by definition, relative and dimensionless. This issue impairs the development of computational models that require that the perceptual scale be translated readily into units of space and time (i.e., centimeters, seconds, etc.).

\section{Visual-Vestibular Methods}

The multisensory nature of self-motion permits investigators to de-couple the relationship among sensory modalities in order to assess each one's contribution to perception. Several investigators have reported the influence of a moving visual surround on an observer's ability to null angular and linear accelerations of the body (Huang \& Young, 1987; Zacharias \& Young, 1981).
In this paradigm, the amount of self-motion induced by the visual surround is measured in terms of the amount of body acceleration, applied in the opposite direction, required to null the perception of self-motion. Body acceleration rather than velocity is reported, because vestibular and other kinesthetic receptors are inertial organs-- meaning that they depend on forces acting on a mass and therefore are insensitive to constant-velocity motions of the body. When a moving visual surround induces self-motion in this paradigm, it introduces a directional bias in the ability to null body motion disturbances. Data from these nulling experiments are variously reported as amount of session completed (a session ends when the observer is unable to null disturbances and as a consequence reaches the stops at the track end) and input-output gain and phase changes. This approach, though not widely employed, has been used to develop computational models of self-motion perception that are directly testable in neurophysiological investigations (see Zacharias \& Young, 1981). One disadvantage of this method is task difficulty; not all observers can perform the task, and even those who can often require extensive training. For example, Huang and Young (1987) reported that only 5 of their $11 \mathrm{ob-}$ servers could complete their experiment. However, this method has the benefit of employing "active" or planned observer behavior and could be used to investigate the contribution of visual surround parameters to vection by examining relative differences in nulling as a function of surround manipulations.

A second type of visual-vestibular paradigm has been employed to measure vection. Several investigators have reported the influence of a moving visual surround on thresholds for detection of inertial self-motion direction (Berthoz, Buizza, \& Schmid, 1977; Berthoz, Pavard, \& Young, 1975; Carpenter-Smith \& Parker, 1992; Lishman \& Lee, 1973; Young, Dichgans, Murphy, \& Brandt, 1973). Reports using these methods consistently reveal that a moving visual surround modulates the intensity of inertial acceleration required to correctly indicate the direction of inertial self-motion.

As for all threshold research, the paradigm involves minimal observer training and is unlikely to present the difficulties of magnitude estimation. In the present study, we chose the $50 \%$ threshold for indicating the direction of inertial self-motion. Specifically, the magnitude of whole-body observer motion that elicits reports of forward self-motion $50 \%$ of the time is designated the point of subjective equality (PSE). Using passive inertial whole-body motion to measure visually induced selfmotion is similar to the active mulling experiments presented earlier, in that the visual and inertial cues are in conflict and the effect of visual surround motion can be described in terms of its impact on decisions about whole-body motion.

\section{Self-Motion Velocity Scaling}

Several investigators (Berthoz et al., 1975; Larish \& Flach, 1990), using magnitude estimation, reported a 
negative exponential relationship between linear vection and visual surround velocity for a given spatial frequency, Lestienne et al. (1977), using postural sway, also reported data to indicate this exponential relationship. Therefore, we chose to investigate the relationship between surround velocity and vection using our method. Specifically, does increasing the velocity of the visual surround increase the magnitude of whole-body motion required to achieve equal numbers of "forward" and "backward" directional reports?

\section{METHOD}

\section{Observers}

Eight students, 1 female and 7 male members of the Miami Spatial Orientation Laboratory staff, participated in the experiment. The observers were between the ages of 20 and 35 years. All the observers were screened for history of equilibrium disorders and had normal or corrected-to-normal vision.

\section{Apparatus}

The Miami University Linear Vection Device, Mark 2 (MULVD2) consisted of a gurney cart and a visual surround made of a pair of endless Mylar belts that were attached to the gurney. The MULVD2 is shown schematically in Figure 1. The device delivered independent linear translational motion of both the observer and the belts. The observers lay in the prone position with the head dorsal-flexed (about $30^{\circ}$ from face-down gaze aligned with the axis of gravity) so that the main parts of the utricular maculae were approximately parallel to the motion. A "cockpit," also attached to the gurney, masked all of the visual field except that revealed by two $46 \times 56 \mathrm{~cm}$ lateral "window" openings that permitted a $60^{\circ} \times 63^{\circ}$ field of view. Eight centimeters behind each opening was a vertically mounted endless Mylar conveyor belt. Each lateral belt was covered with vertical black and white stripes ( $16-\mathrm{cm} / \mathrm{c}$ square wave pattern). The belts were illuminated by incandescent lamps mounted between the cockpit and the belts. The cart movement was produced by a linear dc stepper motor (Compumotor Inc.) that was controlled by a personal computer (Apple Ile). Belt motion was controlled by a variable dc voltage source

\section{Procedure}

Cart motion. In all testing conditions, the observer's whole body was linearly translated either forward or backward from a central resting position. The duration of the body (cart) motion was held constant at $3.5 \mathrm{sec}$, but the amplitude was varied in order to provide a range of peak accelerations. Following each translation, the cart automatically recentered at an acceleration below threshold for direction detection. Representative position, velocity, and acceleration characteristics of this inertial stimulus are shown in Figure 2. The stepper motor that moved the cart was under open-loop control, which produced a motion profile resembling a damped step of position. This stimulus is similar to that used by Benson, Spencer, and Stott (1986). For all conditions, the MULVD2 was programmed so that translations were selected without replacement from a look-up table and were executed in series with a 2 -sec pause between displacements (i.e., after the cart reset, there was a 2 -sec pause before the beginning of the next trial).

Method overview. Several different testing conditions were required in order to investigate the value of passive inertial wholebody motion as a measure of linear vection. First, we conducted a brief period of observer training to be sure that each observer could reliably indicate forward and backward self-motion in darkness. Second, we wanted to quantify each observer's sensitivity to forward and backward whole-body motion in darkness as a baseline measure. To be certain that changes in self-motion reports were attributable to visual surround motion, each observer's sensitivity to whole-body motion was also calculated during exposure to an observer-fixed stationary visual surround (surround velocity $=0 \% \mathrm{sec}$ ). Finally, a series of three different surround velocity conditions $\left(4,14\right.$, and $\left.40^{\circ} / \mathrm{sec}\right)$ were used to measure the change in whole-body motion sensitivity induced by the moving visual surround. Thus, in addition to the initial training, there were five different testing conditions.

Observer training. The observers were translated on a cart in darkness until they were able to report correctly, at the $95 \%$ level, the direction of cart translations at $11.5 \mathrm{~cm} / \mathrm{sec}^{2}$ (approximately twice directional inertial self-motion threshold; Benson et al., 1986) peak acceleration. The observers were instructed to report the direction of the cart motion (i.e., "forward" or "backward") according to the method of constant stimuli (Engen, 1972). They were provided feedback regarding the correctness of each directional response in the training phase only. Masking of background

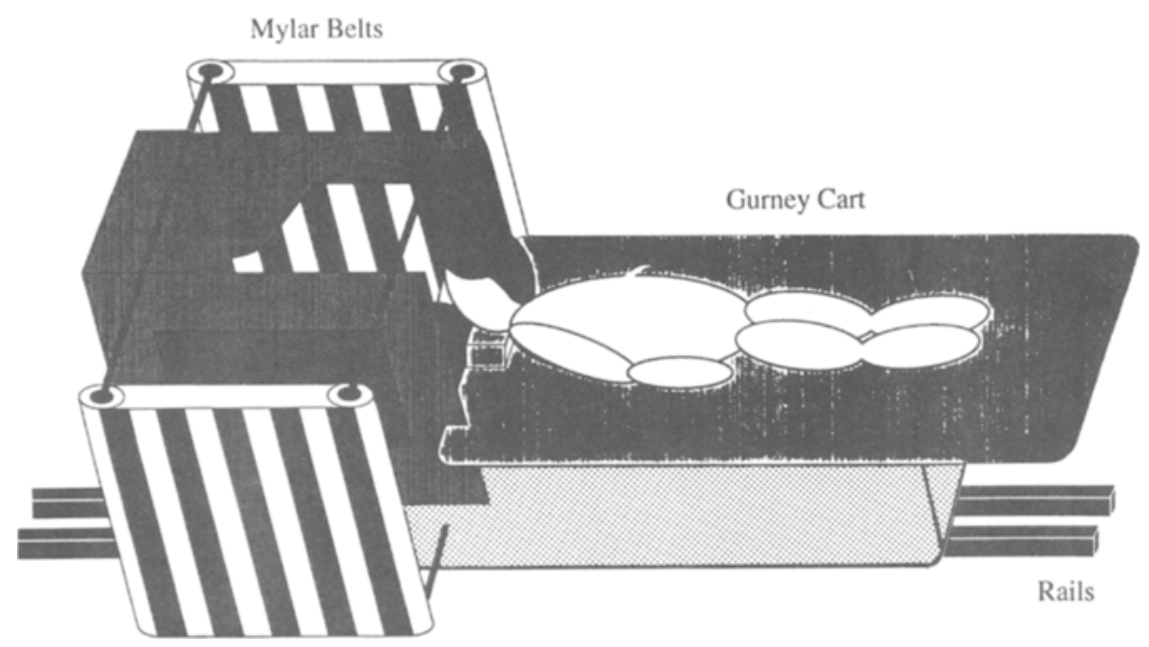

Figure 1. A perspective illustration of the Miami University Linear Vection Device (MULVD2). This device allowed for independent delivery of inertial platform translation and visual surround motion. 
A
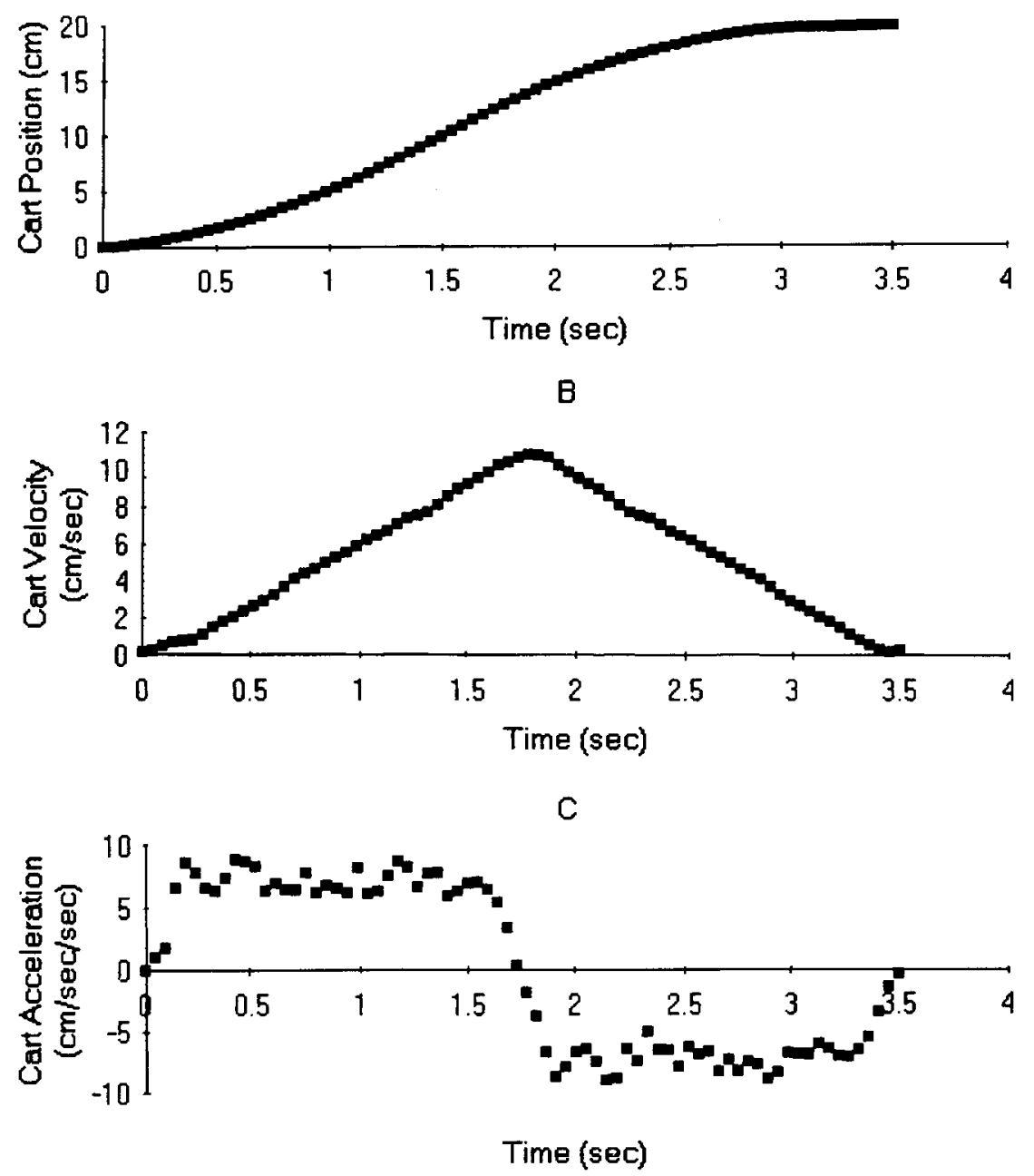

Figure 2. Linear potentiometer recordings of cart position (A), velocity (B), and acceleration to illustrate the inertial stimulus $(C)$. The acceleration values were calculated by using a 3-point difference function and subsequent filtering with a second-order Butterworth LP digital filter.

noise and maintenance of arousal was provided by music presented through headphones.

Dark trials. For each data collection session, the observers were exposed to a series of 60 translations consisting of 10 forward and 10 backward translations at each of three peak acceleration levels presented in pseudorandom order. For each trial, a single tone presented through the headset signaled that the cart would move $3 \mathrm{sec}$ later. The tone also cued the observer to attend to the trial (the observer's vision was occluded). At the conclusion of each cart translation, the tone sounded twice to prompt a verbal report of the direction of completed motion of the cart on which the observer was lying. Acceleration level ranged from 0.5 to $3.0 \mathrm{~cm} / \mathrm{second}$ squared. ${ }^{1}$ Each session consisted of three acceleration levels (i.e., $0.5,1.0,2.0 ; 0.75,1.5,3.0 \mathrm{~cm} / \mathrm{sec}^{2}$ ). Each observer completed five sessions of each set of three acceleration levels in order to have a total of 50 trials at each acceleration level for each direction. The tone sequence was used for all experimental conditions reported in the present paper.

Vision stabilized trials. For each data collection session in the vision stabilized (VS) condition, the visual surround was stationary with respect to the observer. During the VS trials, the visual surround was set at a luminance level controlled by the observer. The observers were instructed to look "straight ahead." As in the dark condition, the observers were exposed to a series of 60 discrete linear translations during each of five data collection sessions.

Vection trials. The procedure during vection was the same as that for the dark and VS trials, with the following exceptions. The visual surround belts moved and were illuminated at a level that the observer reported to be optimal for inducing perceived selfmotion. Four types of trials were presented to each observer in each session:

CF (congruent forward) belt motion simulating forward motion of the observer paired with forward motion of the cart;

$\mathrm{CB}$ (congruent backward) belt motion simulating backward motion of the observer paired with backward motion of the cart;

IF (incongruent forward) belt motion simulating backward motion of the observer paired with forward motion of the cart;

IB (incongruent backward) belt motion simulating forward motion of the observer paired with backward motion of the cart. 
The observers were prompted (by the tone) to open their eyes $3 \mathrm{sec}$ prior to cart translation and to close them when the cart stopped. The directional reports were typically given immediately following the double-tone signal that indicated the end of the displacement. The observers were instructed to look "straight ahead." As in the dark and VS sessions, there were 60 total trials per session. These were divided so that there were $9 \mathrm{CF}, 9 \mathrm{CB}, 21 \mathrm{IF}$, and 21 IB. The observers were informed that congruent as well as incongruent trials would be presented. Higher values of acceleration level were employed in the vection condition relative to the dark and VS conditions because of the dominance of the visual surround on directional reports in the incongruent conditions in pilot tests. In this condition, the peak acceleration ranged from 1.5 to $8.0 \mathrm{~cm} / \mathrm{sec}^{2}$. As in the other conditions, each session consisted of three acceleration levels (i.e., $1.5,3.0,5.75 ; 2.0,4.0,8.0 \mathrm{~cm} / \mathrm{sec}^{2}$ ). Each observer completed seven sessions of each set of three acceleration levels in order to have a total of 49 incongruent and 21 congruent trials at each acceleration level for each direction.

There were six different surround velocity conditions. The average belt velocity (at the observer's eye, computed from the spatial frequency range) was fixed at 4,14 , or $40 \% / \mathrm{sec}$ and traveled either forward or backward with respect to the observer's head. The forward and backward moving surrounds were pseudorandomly presented within a testing session. All the observers were exposed to the dark condition first to obtain a baseline measure of inertial motion sensitivity.

Approximately 62 laboratory testing sessions-each lasting $30-45 \mathrm{~min}$ - were required for an observer to complete all of the conditions in the experiment. To reduce variability, we attempted to use the same observers in all conditions. Due to the extraordinary participation demand, not all of the observers were able to participate in all conditions. Since the same observers participated in many conditions, session order was carefully considered. The order of exposure for subsequent conditions (VS, 4, 14, and $40^{\circ} / \mathrm{sec}$ ) was partially counterbalanced.

\section{Data Analysis}

Calculation of the psychophysical function. The method of data analysis was adapted from the procedure described by Bennett and Westheimer (1985). The psychophysical function relating inertial acceleration to perception of direction for each observer in each condition was determined by pooling the data from all sessions of that condition (e.g., dark, $3.0 \mathrm{~cm} / \mathrm{sec}^{2}$, Observer 4 ) and recording the number of "forward" responses and the number of cart movements for that condition (e.g., 11 "forward" responses from 50 backward cart motions with a peak acceleration of $3 \mathrm{~cm} / \mathrm{sec}^{2}$ for Observer 4 in the dark condition). For each observer and each stimulus condition, the number of "forward" responses for each cart acceleration was then entered into a bootstrapping program developed explicitly for estimating parameters from small data sets (Foster \& Bischof, 1991).

The bootstrapping algorithm computed estimates of the point of subjective equality (PSE; $50 \%$ forward responses) and the slope of the psychometric function. For each function, a chi-square test for goodness of fit was computed. Only psychometric data with significant chi-square values $(p<.05)$ were reported (this rejection criteria was met twice).

\section{RESULTS}

Psychometric data from 1 observer are presented in Figure 3 . The figure includes the psychophysical function relating proportion of "forward" responses to peak inertial acceleration during exposure to three stimulus conditions: VS (i.e., belt velocity $=0 \% \mathrm{sec}$ ), belts mov- ing forward at $40^{\circ} / \mathrm{sec}$, and belts moving backward at $40^{\circ} / \mathrm{sec}$. For this observer, the PSEs were as follows: $-0.34 \mathrm{~cm} / \mathrm{sec}^{2}$ for the VS condition, $-5.04 \mathrm{~cm} / \mathrm{sec}^{2}$ for the forward-belt condition, $2.30 \mathrm{~cm} / \mathrm{sec}^{2}$ for the backward-belt condition, and $-0.08 \mathrm{~cm} / \mathrm{sec}^{2}$ for the dark condition (not shown in Figure 3). In addition to the PSE values, the slope of each function may provide further information about the effects of the different surround conditions. This issue is not addressed in this paper, but may be investigated in future research.

The mean PSE data from all of the observers for the four belt velocities ${ }^{2}\left(0,4,14\right.$, and $\left.40^{\circ} / \mathrm{sec}\right)$ are presented in Figure 4 . These data were fit by a linear regression equation that revealed that about $94 \%$ of the variance was accounted for by the relationship between the belt velocity and mean PSE. Although a first-order regression provides a good fit for the data, this does not preclude other higher order, nonlinear models. Note too that the function is symmetrical and passes through the coordinate 0,0 .

PSE data for each subject in each condition are presented in Table 1. A multiple regression analysis was computed for the pooled signed data of the 8 observers. There was a significant effect of surround condition $[F(7,57)=10.92, p<.0001]$. For all analyses, we chose the more conservative between subjects design analysis, even though most of the same subjects participated in each condition.

Duncan's multiple-range test for the PSE values was calculated to permit post hoc comparisons between visual surround condition means. The differences between the mean PSEs in the dark and VS conditions were not significantly different $(p>.05)$. The different belt motion condition PSE means showed some directional asymmetries. For example, the forward-belt velocities of 4 and $14^{\circ} / \mathrm{sec}$ yielded PSEs that were not statistically different from those for the dark or VS condition. However, both the 4 and $14 \%$ sec backward-belt velocities elicited PSEs that were statistically different from those for the dark and VS conditions.

To determine whether the magnitude of forward vection was equal to the magnitude of backward vection, a multiple regression analysis was computed for the absolute value of PSE. There was a significant effect of the surround condition $[F(7,57)=8.62, p<.0001]$. However, the post hoc analysis of the PSEs for the direction of belt motion within each moving surround condition (e.g., forward and backward $4 \%$ sec surround motion) indicated that there were no statistically significant differences in the magnitude of the mean PSE for each direction of belt motion at each surround velocity.

\section{Subjective Reports}

The observers volunteered reports of fluctuations between self-motion and surround motion. These changes in state occurred intermittently throughout all of the vection conditions. These alternations between visually induced self-motion and self-stationarity with respect to a 


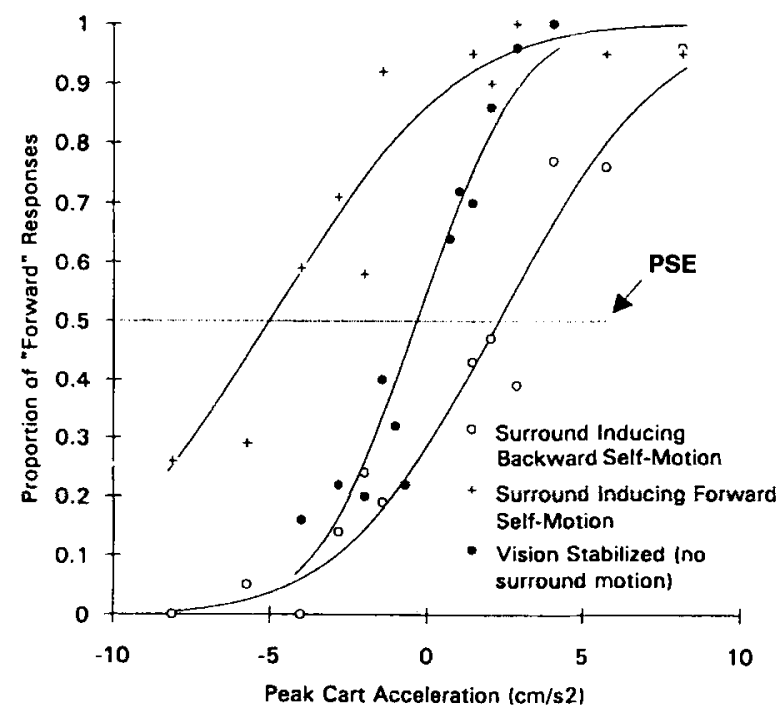

Figure 3. Proportion of "forward" responses elicited from a typical subject during exposure to the vision stabilized and $40 \% \mathrm{sec} \mathrm{mov-}$ ing visual surround conditions. The point of subjective equality (PSE) is indicated by the dashed horizontal line.

moving visual field have been described for circular vection by Held, Dichgans, and Bauer (1975) and Mergner and Becker (1990).

\section{DISCUSSION}

The demonstration of a directional shift in the PSE in sensitivity to inertial acceleration induced by exposure to a moving visual surround is evidence that we have developed a new measure of linear vection. The finding that the PSEs obtained in the moving surround conditions were significantly different from those obtained in the dark or VS condition suggests that the magnitude and direction of inertial self-motion perception was influenced by the visual surround. For example, if the surround had not induced motion of the observer, it is not likely that, by chance, the PSE would shift away from VS levels with such directional consistency (i.e., forward-inducing surround moved elevated sensitivity to backward motion). The combined PSE data presented in Figure 4 suggest that linear vection is monotonically related to surround velocity. To further support the validity of the present method, the regression equation fitting the data in Figure 4 intersects a PSE value of 0.0 for the VS condition. Thus, when the visual surround is not moving, the PSE is aligned with zero cart acceleration.

Another interesting aspect of Figure 4 is the apparent equivalent slopes of the forward and backward PSEs. This suggests that sensitivity to forward and backward vection is similar. This finding is echoed in the multiple regression analysis of the absolute value of PSE, in which no significant differences were found in the mag- nitude of the forward and backward PSE level at each belt velocity.

The finding that vection increases monotonically with surround velocity is congruent with previous reports using similar surround velocities but different psychophysical methods. Recall that Berthoz and his colleagues (Berthoz et al., 1975) reported a negatively accelerating curve relating surround velocity to self-motion perception. The present investigation employed a constant-velocity visual surround motion that was fixed relative to the subject. This differs from the accelerating visual surround used by Berthoz et al. (1975) as well as the observer-relative acceleration of visual surround motion that must occur during postural sway when the surround is not fixed to the subject (Lestienne et al., 1977). Although there were differences in the nature of the moving surround, the relationship between surround velocity and vection was similar. It would be interesting to see whether active nulling yields monotonic increases with similar growth when multiple surround velocities are compared.

The reports volunteered by the subjects during data collection included a "switching" between perception of self-motion with respect to a stationary visual surround, and of a stationary self with respect to a moving surround. Held et al. (1975) and, more recently, Mergner and Becker (1990) have discussed this bistability as it relates to circular vection. Since our investigation is limited to inferences derived from directional reports, it can be assumed that such bistability would bias our data to underestimate the shift in PSE. For example, if during the cart motion the subject perceived the surround motion as surround motion (instead of self-motion with respect to a stable surround), reports of cart direction would tend toward levels of sensitivity observed in the dark or VS conditions. Future investigation of these phenomena should include posttranslation indications of perceptual state (e.g., "I felt like the surround was stationary," or "I felt like the surround was moving"; see Held et al., 1975).

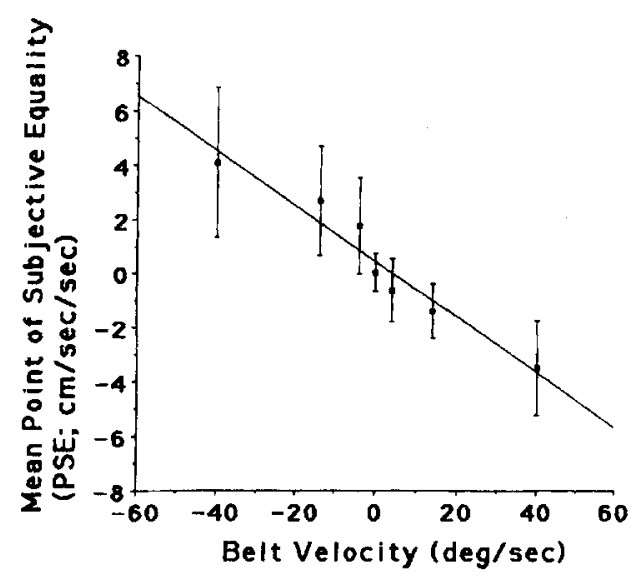

Figure 4. The mean and standard error of PSE as a function of average visual surround velocity. 
Table 1

Point of Subjective Equality $\left(\mathrm{cm} / \mathrm{sec}^{2}\right)$ for Each Subject in All Experimental Conditions

\begin{tabular}{|c|c|c|c|c|c|c|c|c|}
\hline \multirow[b]{2}{*}{ Subject } & \multicolumn{8}{|c|}{ Surround Motion Condition } \\
\hline & Dark & -40 & -14 & -4 & 0 & 4 & 14 & 40 \\
\hline 1 & -0.56 & 10.25 & 4.25 & 3.58 & 0.34 & -2.41 & -3.2 & -3.70 \\
\hline 2 & 0.75 & 4.53 & 0.47 & 0.08 & 0.37 & 0.28 & -0.0 & -4.95 \\
\hline 3 & -0.15 & 6.20 & 5.22 & 4.32 & 1.41 & -2.25 & -1.7 & -4.43 \\
\hline 4 & -0.24 & 0.89 & 0.76 & 0.30 & 0.28 & -1.18 & -0.7 & -.032 \\
\hline 5 & -0.17 & 1.32 & 0.86 & $\mathrm{~N} / \mathrm{A}$ & -0.11 & $\mathrm{~N} / \mathrm{A}$ & -1.4 & -0.92 \\
\hline 6 & -0.08 & 2.30 & $\mathrm{~N} / \mathrm{A}$ & 1.08 & -0.34 & 0.56 & N/A & -5.04 \\
\hline 7 & 0.33 & 5.09 & 4.43 & 3.79 & -0.53 & 0.23 & -2.32 & -2.30 \\
\hline 8 & 0.20 & * & 0.12 & 0.26 & -0.76 & 0.45 & 0.46 & $*$ \\
\hline \multirow[t]{3}{*}{$M$} & 0.03 & 4.37 & 2.30 & 1.75 & 0.01 & -0.61 & -1.31 & -3.09 \\
\hline & A & & & & A & A & A & \\
\hline & & & B & B & & & & \\
\hline
\end{tabular}

\begin{abstract}
Note-Numeric surround conditions reflect the average velocity and direction of surround motion. Negative values indicate surround motion that induces backward self-motion, and positive values indicate surround motion that induces forward self-motion perception. The " 0 " surround condition refers to the vision stabilized trials. Any two means with the same letter are not significantly different $(p>.05)$. N/A indicates that the subject was not available to participate. *Data from this subject were associated with a nonsignificant $(p>.05)$ chi-square value for the bootstrapping calculations.
\end{abstract}

In the present investigation we have demonstrated the value of inertial acceleration as a mechanism to scale visually induced perceptions of self-motion. One question that has been raised is: Why compare a visual velocity to an inertial acceleration? Other investigators have provided data that suggest the presence of integrators in the nervous system that transform inertial acceleration information into velocity and position information (Israel \& Berthoz, 1989; Melvill Jones \& Young, 1978; Young \& Meiry, 1968). Therefore, at the level of the central nervous system, visual velocity may be compared with the velocity component of the inertial acceleration. Indeed, there is ample neurophysiological evidence demonstrating the equal sufficiency of vestibular and visual afferents stimulation in eliciting activity of neurons in the vestibular nuclei of monkeys and rats (Horn, Miller, \& Neilson, 1983; Waespe \& Henn, 1979). We chose to express vection in terms of PSE acceleration in order to be consistent with the majority of the previous linear self-motion literature (reviewed in Howard, 1986) and to be clear that a constant-velocity whole-body motion is not a stimulus to the vestibular or somatosensory receptors.

One issue that was not addressed in the present investigation is the possibility of adaptation of self-motion perception. The present method required that the subject report the direction of cart (body) motion according to the method of constant stimuli. Visually induced selfmotion is inferred from any systematic change in the PSE. Although the subjects in the present study closed their eyes between cart translations, any changes in sensitivity to vection that might occur within the $4-5 \mathrm{sec}$ that the observer had his/her eyes open would serve to reduce any influence on PSE. Such adaptation could be assessed through manipulation of the period of the cart motion and/or exposure to the moving surround.
The present investigation was confined to the case of linear vection, but the general method can be easily adapted for circular vection. Future research should address the bistable nature of linear vection, which previously has not been reported or discussed.

\section{Conclusions}

The following conclusions derive from this investigation:

1. Linear vection can be measured as the amount of whole-body motion required to elicit equal numbers of "forward" and "backward" reports of self-motion direction.

2. Our data suggest that linear vection increases monotonically with surround velocities between 0 and $40^{\circ} / \mathrm{sec}$.

\section{REFERENCES}

Andersen, G. J., \& Dyre, B. P. (1989). Spatial orientation from optic flow in the central visual field. Perception \& Psychophysics, 45, 453-458.

Bennett, R. G., \& Westheimer, G. (1985). A shift in the perceived simultaneity of adjacent visual stimuli following adaptation to stroboscopic motion along the same axis. Vision Research, 25, 565-569.

Benson, A. J., Spencer, M. B., \& Stott, J. R. R. (1986). Thresholds for the detection of direction of whole-body, linear movement in the horizontal plane. Aviation, Space, \& Environmental Medicine, 57, 1088-1096.

Berthoz, A., BuizzA, A., \& Schmid, R. (1977). Visual-vestibular interaction during linear motion. Proceedings of the European Symposium on Life Sciences Research in Space (ESASP-130, pp. $117-$ 125). Cologne: The European Space Agency.

Berthoz, A., \& Droulez, J. (1982). Linear self-motion perception. In H. Wertheim, A. Wagenaar, \& W. Leibowitz (Eds.), Tutorials on motion perception (pp. 157-199). New York: Plenum.

Berthoz, A., Pavard, B., \& Young, L. R. (1975). Perception of linear horizontal self-motion induced by peripheral vision (linear vection): Basic characteristics and visual vestibular interaction. Experimental Brain Research, 23, 471-489. 
BrindLey, G. S. (1970). Physiology of the retina and visual pathway: New York: Williams \& Wilkins.

Carpenter-Smith, T. R., \& Parker, D. E. (1992). The effects of unidirectional visual surround translation on detection of physical linear motion direction: A psychophysical scale for vection. In B. Cohen, D. Tomko, \& F. Guedry (Eds.), Sensing and controlling motion: Vestibular and sensorimotor function (Annals of the New York Academy of Sciences, Vol. 656, pp. 817-819). New York: New York Academy of Sciences.

Dichgans, J., \& BRANDT, T. (1978). Visual-vestibular interaction: Effects on self-motion perception and postural control. In R. Held, H. Leibowitz, \& H.-L. Teuber (Eds.), Handbook of sensory physiologv: Vol. 8. Perception (pp. 756-804). New York: Springer-Verlag.

EngEN, T. (1972). Psychophysics II: Scaling methods. In J. W. Kling \& L. A. Riggs (Eds.), Experimental psychology: Vol. I. Sensation and perception (pp. 47-79). New York: Holt, Reinhan \& Winston.

Fischer, M. H., \& Kornmuller, A. E. (1930). Optokinetisch ausgeloste Bewegungswahrnehmungen und optokinetischer Nystagmus [Optokinetic induced motion perception and optokinetic nystagmus]. Journal für Psychologie und Neurologie, 41, 273-308.

Foster, D. H., \& Bischof, W. F. (1991). Thresholds from psychometric functions: Superiority of bootstrap to incremental and probit variance estimators. Psychological Bulletin, 109, 152-159.

Held, R., Dichgans, J., \& Bauer, J. (1975). Characteristics of moving visual scenes influencing spatial orientation. Vision Research, 15, 357-365.

Horn, K. M., Miller, S. W., \& Neilson, H. C. (1983). Visual modulation of neuronal activity within the rat vestibular nuclei. Experimental Brain Research, 52, 311-313.

Howard, I. P. (1986). The perception of posture, self-motion, and the visual vertical. In K. R. Boff, L. Kaufman, \& J. P. Thomas (Eds.), Handbook of perception and human performance: Vol 1. Sensory processes and perception (pp. 18-1 to 18-62). New York: Wiley.

HowarD, I. P. (1989). Spatial vision within egocentric and exocentric frames of reference. In S. R. Ellis, M. K. Kaiser, \& A. Grunwald (Eds.), Spatial displays and spatial instruments (NASA Conference Publication 10032, pp. 10-1 to 10-17). Moffett Field, CA.

Huang, J.-K., \& Young, L. R. (1981). Sensation of rotation about a vertical axis with a fixed visual field in different illuminations and in the dark. Experimental Brain Research, 41, 172-183.

HUANG, J.-K., \& YounG, L. R. (1987). Influence of visual and motion cues on manual lateral stabilization. Aviation, Space, \& Environmental Medicine, 58, $1197-1204$.

IsRAEL, I., \& Berthoz, A. (1989). Contribution of the otoliths to the calculation of linear displacement. Journal of Neurophysiology, 62, 247-263.

LARISH, J. F., \& FLACH, J. M. (1990). Sources of optical information useful for perception of speed of rectilinear self-motion. Journal of Experimental Psychology: Human Perception \& Performance, 16, 295-302.

LEE, D. N., \& Aronson, E. (1974). Visual proprioceptive control of standing in human infants. Perception \& Psychophysics, 15, 529-532.

Lestienne, F., Soechting, J., \& Berthoz, A. (1977). Postural readjustments induced by linear motion of visual scenes. Experimental Brain Research, 28, 363-384.

LishmaN, J. R., \& LEE, D. N. (1973). The autonomy of visual kinestheses. Perception, 2, 287-294.

MACH, E. (1875). Grundlinien der Lehre von den Bewegungsempfin- dungen [Outline for the theory of motion sensation]. In R. Held. H. Leibowitz. \& H.-L. Teuber (Eds.). Handhook of sensom phisiology: Vol 8. Perception (pp. 756-804). New York: Springer-Verlag

Melvil. Jones, G., \& Young, L. R. (1978). Subjective detection of vertical acceleration: A velocity-dependent response'? Acta Otolaryngologica, 85, 45-53

Mergner, T. \& Becker, W. (1990). Perception of horizontal selfrotation: Multisensory and cognitive aspects. In R. Warren \& A. H Wertheim (Eds.), Perception and control of self-motion (pp. 219 263). Hillsdale, NJ: Erlbaum.

Poulton, E. C. (1968). The new psychophysics: Six models for magnitude estimation. Psychological Bulletin, 69, 1-19.

Schmidt, F., \& Tiffin, J. (1969). Distortion of drivers" estimates of automobile speed as a function of speed adaptation. Journal of Applied Psychology, 53, 536-539

STEVENS, S. S. (1961). Psychophysics of sensory function. In W. Rosenblith (Ed.), Sensory communication (pp. 36-52). Cambridge, MA MIT Press.

Stoffregen, T. A. ( 1985). Flow structure versus retinal location in the optical control of stance. Journal of Experimental Psychology: Human Perception \& Performance, 11, 554-565.

Teghtsoonian, R. (1971). On the exponents in Stevens' law and the constants in Ekman's law. Psychological Review, 78, 71-80.

WAESPE, W., \& HENN, V. (1979). The velocity response of vestibular nucleus neurones during vestibular, visual, and combined angular acceleration. Experimental Brain Research, 37, 337-347.

Warren, W. H., \& KurTz, K. J. (1992). The role of central and peripheral vision in perceiving the direction of self-motion. Perception \& Psychophysics, 51, 443-454.

Young, L. R., Dichgans, J., Murphy, R., \& Brandt, T. (1973). Interactions of optokinetic and vestibular stimuli in motion perception. Acta Otolaryngologica, 76, 24-31.

YOUNG, L. R., \& MEIRY, J. L. (1968). A revised dynamic otolith model. Aerospace Medicine, 39, 606-608.

ZaCharias, G. L., \& YoUnG, L. R. (1981). Influence of combined visual and vestibular cues on human perception and control of horizontal rotation. Experimental Brain Research, 41, 159-171.

\section{NOTES}

1. The values for peak cart acceleration were originally determined by using a decibel scale $\left(0 \mathrm{~dB}=0.001 \mathrm{~cm} / \mathrm{sec}^{2}\right)$. Observers were presented with cart motions that were in 6-dB increments in order to obtain a wide range of inertial stimuli. For graphic and analytical clarity, the actual values of peak acceleration were used for all analyses.

2. Although the PSE values are presented in relation to average surround velocity, the constant surround spatial frequency pattern produced temporal frequencies (TF) that were proportional to the surround velocity. To calculate spatial and temporal frequency, the present constant-velocity stimulus would need to be described in terms of its retinal projection. On the retina, the horizontal spatial frequencies ranged between 0.03 and $0.09 \mathrm{c} / \mathrm{deg}$, projected on the observer's eye. Therefore, the data could have been plotted with PSE magnitudes as a function of TF range, and the same graphic relationships would result.

(Manuscript received November 29, 1993; revision accepted for publication July 26, 1994.) 